\title{
Teoría industrial:
}

un vínculo con

el clúster del calzado*

/ Industrial theory:

a link with the cluster

of footwear

* Recibido: 17 de octubre de 2016. Aceptado: 23 de diciembre de 2016.

Tla-Melaua, revista de Ciencias Sociales. Facultad de Derecho y Ciencias Sociales.

Benemérita Universidad Autónoma de Puebla, México / Isss: 1870-6916 / Nueva Época, año 11, núm. 42, abril-septiembre 2017, pp. 116-135. 
Este trabajo tiene como objetivo analizar las fuerzas de atracción que llevaron a la región mexicana de León, Guanajuato, a convertirse en un ejemplo de desarrollo regional, a partir de la industria del calzado. La metodología parte del análisis de la teoría industrial, particularmente la localización, la aglomeración y el clúster industrial. Es una investigación de contraste; vincula un fenómeno teórico con una investigación empírica. Determina las causas empíricas por las cuales se generó la localización y aglomeración industrial y en qué condiciones. Para ello, se elaboraron entrevistas a los empresarios del calzado.

PALABRAS CLAVE

Localización, aglomeración indus- Location, industrial agglomeration, trial, industria del calzado, León, footwear industry, León, Guanajuato. Guanajuato.
This research paper aims to analyze the forces of attraction that led the Mexican region of Leon, Guanajuato, to become an example of regional development, based on the footwear industry. The methodology starts from the analysis of the industrial theory, particularly the localization, the agglomeration and the industrial assemblage. It is a research of contrast; it contrasts a theoretical phenomenon with an empirical research. It determines the empirical causes by which the localization and industrial agglomeration was generated and what were the conditions. Interviews were made to the footwear entrepreneurs in order to gather the required information.

KEYWORDS

\footnotetext{
* Profesor investigador en el Colegio del Estado de Hidalgo, México. (maximiliano@elcolegiodehidalgo.edu.mx)
} 
1. Introducción / 2. Localización y aglomeración: distrito industrial y clúster / 3. Origen, evolución e importancia de la industria de calzado en León / 4. Metodología para la investigación en el estudio de caso / 5. La industria del calzado en León. Vinculación entre lo teórico y lo empírico / 6. Reflexiones finales

\section{INTRODUCCIÓN ${ }^{1}$}

En los últimos años ha renacido el interés por estudiar la localización de las actividades industriales y el éxito que éstas han tenido en los países de ingresos altos, particularmente cuando se logran conformar clústeres industriales. Algunos casos de éxito que comprueban lo anterior son: la electrónica, en Silicon Valley; la industria del automóvil, en Detroit; la industria de cerámica, en Sassuolo; el calzado, en Brenta, Elche y León; la industria textil-confección, en Reutlingen, Santo Domingo y Pekín.

A partir de los resultados obtenidos en el trabajo de campo, el objetivo de esta investigación es determinar si la localización, evolución y consolidación de la industria del calzado en León se vincula con la teoría de la localización, de la aglomeración y de los clústeres industriales.

La investigación desarrolla una conexión entre la parte teórica, con un trabajo de campo realizado a empresarios de calzado en León, ${ }^{2}$ Guanajuato; México. Se realizaron dos tipos de estudios: el primero, exploratorio y el segundo, descriptivo. La parte exploratoria permite desarrollar una investigación con visión general de tipo aproximativo, respecto a la realidad de la industria del calzado en León. La parte descriptiva especifica las características de la industria de estudio y los servicios vinculados a ésta. Además, se relaciona con la primera parte del trabajo, en la cual se revisa la teoría de la localización, la aglomeración y los clústeres industriales. Todos éstos son elementos teóricos que permiten comprender el origen, la evolución y consolidación de la industria de calzado en León.

Para alcanzar el objetivo antes señalado, el trabajo presenta la siguiente estructura: en la primera parte se describe la introducción del texto; en el segundo apartado se presenta el enfoque teórico; la tercera sección explica

\footnotetext{
${ }^{1}$ Este trabajo enriquece una versión preliminar denominada: La industria del calzado en León, Guanajuato; México. Análisis a partir de las economías externas y de urbanización. Disponible en: http://www. academia.edu/10826250/la_industria_del_calzado_en_le\%c3\%93n_guanajuato_m\%c3\%89xico._. an\%c3\%81lisis_a_partir_de_las_econom \%c3\%8das_externas_y_de_urbanizaci\%c3\% $\% 3 \mathrm{n}$

${ }^{2}$ A partir de aquí le llamaremos León. Su nombre oficial es León de los Aldamas, se ubica en el centro de México, a 360 kilómetros de la Ciudad de México (capital de la república mexicana).
} 
el origen del calzado en León y su importancia económica para dicho municipio; la cuarta parte presenta la metodología utilizada en el trabajo de campo; la última sección, además de ser la más importante, vincula los aspectos teóricos con los empíricos, ello a partir de un trabajo de campo esquematizado con la aplicación de entrevistas a empresarios del calzado en el municipio de León.

Con la estructura antes señalada, se detecta la situación actual del sector de estudio. Pero, lo principal y más importante de esta investigación fue descubrir si existe una relación entre la parte teórica y la empírica dentro del fenómeno estudiado.

\section{LOCALIZACIÓN Y AGLOMERACIÓN: DISTRITO INDUSTRIAL Y CLÚSTER}

Alfred Marshall efectuó el primer análisis de las economías de localización. Estudió los fenómenos por los cuales las empresas de un sector encuentran ventajas cuando se concentran en el territorio. Con ello, observan rendimientos crecientes para el conjunto de la agrupación empresarial, aunque no para cada empresa en particular. Lo anterior implica revisar las condiciones dadas en el territorio para el nacimiento y consolidación de una industria en una localización concreta. Al respecto, Alfred Marshall argumenta:

Cuando una industria ha escogido una localidad para situarse en ella, es probable que permanezca en la misma durante largo tiempo, pues son muy grandes las ventajas que los que se dedican a la misma industria obtienen de la misma proximidad. ${ }^{3}$

Si se analiza no sólo el surgimiento, sino también la expansión de una actividad industrial, se deben considerar las condiciones de producción de las actividades relacionadas. De ahí surgen las economías externas, es decir, las economías de producción a gran escala, las cuales raramente pueden ser atribuidas a una industria en particular. Están en gran medida conectadas con grupos, frecuentemente grandes grupos de industrias correlacionadas ${ }^{4}$.

Para Marshall las economías externas son factores que influyen en los costos o en la función de producción, los cuales no se originan en el tamaño de cada empresa individual, sino en el conjunto del distrito industrial. ${ }^{5}$ Las

\footnotetext{
${ }_{3}^{3}$ Marshall, Alfred, Principles of Economics, London, Macmillan, p. 156. [Consulta: 28 de marzo, 2016]. Disponible en: http://files.libertyfund.org/files/1676/Marshall_0197_EBk_v6.0.pdf

${ }^{4}$ Ibidem, pp. 184-185.

${ }^{5} \mathrm{El}$ distrito industrial se puede definir como áreas mono productoras donde las empresas mantienen interrelaciones de sentido horizontal, sobre todo de subcontratación, combinando de forma aún incipiente competencia y colaboración, en redes progresivamente densas y con un mayor protagonismo de las instituciones locales en la generación de iniciativas de promoción y desarrollo. Méndez, Ricardo, Caravaca, Inmaculada, Organización industrial y territorio, Madrid, Síntesis.
} 
economías externas permiten a Marshall demostrar que es posible alcanzar economías de escala en función del tamaño productivo de un sistema de empresas localizadas en un territorio, y no de la dimensión particular de cada empresa. De ahí surge la importancia de la localización y posterior aglomeración industrial de la industria de calzado en León.

Para Marshall, un sistema de pequeñas empresas especializadas en distintas funciones de producción responde a la división del trabajo y es resultado de la ampliación del tamaño del mercado. Esto beneficia a la sociedad, dada la flexibilidad en las relaciones de las empresas, lo cual estimula la generación de innovaciones.

Marshall dividió en tres grupos a los factores que pueden generar externalidades y, por tanto, estimular la concentración de las empresas de un sector en un determinado territorio. ${ }^{6}$

- El primer grupo es el de los flujos de información, los cuales están relacionados con las habilidades y los conocimientos específicos del sector. Los flujos de información se difunden fácilmente entre las empresas cercanas, se genera así un proceso acumulativo de saber hacer. Marshall fue quien por primera vez identificó la importancia de los desbordamientos en la concentración de las actividades en el territorio.

- Segundo: cuando las empresas de un sector se aglomeran, generan el establecimiento de otras actividades que son complementarias. Esto significa proveedoras de entradas y servicios que generan beneficios al sector. Un lugar donde se ubiquen diferentes empresas les permite tener acceso a diferentes factores de producción, a menores costos.

- Tercero: cuando se aglomeran las empresas, se forma un mercado de trabajo especializado, por tanto, se puede compartir con todas las empresas del sector. Trabajadores y empresarios ganan con la aglomeración. Para el empresario representa una oferta de trabajo especializado, mientras los trabajadores tienen la seguridad de no depender solamente de una empresa para obtener un empleo. Esto significa que cualquier industria concentrada puede ofrecer un mercado de mano de obra, lo cual genera la atracción de trabajadores hacia ese lugar en busca de empleo.

Los elementos básicos de la teoría de Weber son los factores de aglomeración, los cuales están constituidos por circunstancias que abaratan la producción y distribución por realizarse en un solo lugar. Para Weber, los factores de

\footnotetext{
${ }^{6}$ Ibidem, pp. 154-160.
} 
aglomeración son "resultado de la naturaleza social de la producción, por lo que no pueden ser descubiertos analizando procesos productivos aislados". ${ }^{7}$

La vinculación de las empresas impulsa las ventajas de la aglomeración atrayendo nuevas empresas en un proceso que, en forma continua, alcanza etapas superiores de desarrollo tecnológico, organización laboral e impulso de la organización económica, derivada de factores de mercado y descenso de los costos generales por establecimiento. ${ }^{8}$

Alfred Weber considera que las economías de aglomeración son un factor determinante de la localización industrial. Sin embargo, su definición se restringe a la de economías de escala generadas por la presencia de multi productos, que pueden establecer nexos intersectoriales.

Weber y Marshall son autores obligados dentro de los estudios originales de la localización y la aglomeración industrial. No obstante, otros autores han enriquecido las primeras aportaciones teóricas, por lo cual debemos ir más allá y considerar autores contemporáneos de la misma línea de investigación. En ese sentido, Krugman señala que tanto las condiciones históricas como el nivel de los costos de transporte son importantes en las decisiones de localización. Krugman matiza que

Lo que da lugar a un vínculo hacía atrás significativo, en el sentido de Hirschman, no es sólo el hecho de que el sector A compre el output del sector B; es la afirmación de que la inversión en A, aumenta el volumen del mercado de $\mathrm{B}$, lo cual induce a un cambio de escala en la producción de B que resulta más eficiente [...] Quizá B ya esté en una escala eficiente, o quizá esta expansión no lo acerque a esa escala [...] Si uno trata de utilizar la retórica de los vínculos, sin entender que es un argumento que depende decisivamente de las economías de escala, puede acabar diciendo disparates. ${ }^{9}$

Krugman y Elizondo ${ }^{10}$ argumentan que las empresas tienden aproximarse al mercado más grande, es decir al lugar donde se localiza la mayoría de sus clientes. Esta tendencia corresponde al vínculo de demanda (fordward linkage). También buscan aproximarse a sus abastecedores de insumos, conjunto que incluye sobre todo a los productores de bienes de consumo utilizados por el insumo fuerza de trabajo. Esta segunda fuerza de atracción corresponde al

\footnotetext{
${ }^{7}$ Weber, Alfred, Theory of the Location of Industries. Chicago, 1929, p. 125. [Consulta: 18 de marzo, 2016]. Disponible en: https://archive.org/stream/alfredweberstheo00webe\#page/n25/mode/2up ${ }^{8}$ Ibidem, pp. 124-174.

${ }^{9}$ Krugman, Paul, "Growing world trade: causes and consequences", Brookings Papers on Economic Activity, núm. 1, p. 23.

${ }^{10}$ Krugman, Paul \& Elizondo, Raúl Livas, "Trade policy and the Third World metropolis", Journal of Development Economics, núm. 1, pp. 137-150.
} 
vínculo de costos (backward linkage). Para estos autores, cuanto mayor es el número de empresas que hay en un lugar, menor es el precio asociado a la localización (vínculo de costo), por ende, se tiene que mejorar dentro de la competencia internacional.

Para Krugman "La aglomeración plantea un modelo interesante de geografía económica que recoge la tensión entre dos tipos de fuerzas: las fuerzas centrípetas, que tienden a empujar la actividad económica a integrarse en aglomeraciones, y las fuerzas centrífugas, que tienden a romper esas aglomeraciones o a limitar su tamaño". ${ }^{11}$

A causa de los rendimientos crecientes, es preferible concentrar la actividad y la producción de cada producto en unas pocas ubicaciones. Por los costos de transporte, las mejores localizaciones son aquellas que tienen un buen acceso a los mercados (vinculación hacia delante) y a los proveedores (vinculación hacia atrás). Pero el acceso a los mercados y a los proveedores está precisamente en los puntos en los que se han concentrado las fábricas y que, en consecuencia, han atraído a sus alrededores a los factores de producción móviles. Pero no todos los factores son móviles, y la presencia de los factores inmóviles es lo que da lugar a la fuerza centrífuga que actúa en contra de la aglomeración. ${ }^{12}$

Para los autores Baldwin y Venables, un proceso de aglomeración necesita vínculos positivos entre las actividades de los agentes que operan en una misma localización. Estos vínculos pueden ser externalidades tecnológicas, las cuales tienen un alcance espacial limitado, o externalidades pecuniarias, las cuales pueden surgir de una industria que opera en competencia imperfecta.

Estos autores también señalan la diferencia entre vínculos de demanda y vínculos de costo. Argumentan que si un incremento en el número de empresas de una localización genera un incremento en las ganancias de las otras empresas situadas en aquella localización, a través de un aumento de la demanda de sus productos, se presentarán vínculos de demanda. Por otra parte, si el aumento de los beneficios de las otras empresas se corresponde con una reducción en el costo de los inputs, derivada de la entrada de nuevas empresas, aparecen entonces los vínculos de costos.

Para Porter, ${ }^{13}$ muchos análisis de las economías de aglomeración destacan la reducción de costos como resultado de la proximidad a los factores de producción o la proximidad de los mercados. Pero, para él, estas explicaciones

\footnotetext{
${ }^{11}$ Krugman, op. cit.

${ }^{12}$ Ibidem, pp. 87-88.

${ }^{13}$ Porter, Michael, La ventaja competitiva de las naciones, Buenos Aires, Javier Vergara, 1991, p. 219.
} 
se han visto rebasadas por la globalización que ha traído la mundialización de los mercados, las tecnologías y las fuentes de suministros, por la movilidad y reducción de los costos de transporte. Para el autor el nivel actual de las economías de aglomeración es el de los clústeres.

Michael Porter contrapone el concepto de ventaja comparativa al de ventaja competitiva de las naciones. Reconoce a la empresa y sus sectores de competencia como las unidades básicas en que operan las ventajas competitivas. Así, se desarrolla también el concepto de competencia, la cual incluye un conjunto de mercados segmentados, productos diferenciados, diferencias tecnológicas y de economías de escala.

Para este autor, lo que hace próspero a un país es la capacidad de sus empresas para alcanzar elevados niveles de productividad, es decir, la capacidad de usar con eficiencia y creatividad la mano de obra, los recursos naturales y el capital. Para Porter, no importa cuánto se posea, sino qué se hace con lo que se tiene, y esto lo subraya constantemente.

No es el mero acceso a los factores, sino la capacidad de desplegarlos productivamente lo que tiene una gran importancia para la ventaja competitiva. Ésta es resultado de la forma en que las empresas organizan las diferentes actividades que componen su cadena de valor y del grado de coordinación entre todas ellas. Para lograrlo, debe realizar sus actividades de forma más eficiente que sus competidores. ${ }^{14}$

Porter señala que las empresas tienen varias opciones para incrementar su competitividad. Por una parte, tienen el poder de competir en precio mediante el mecanismo de abaratar costos, lo cual se logra a través de:

- Las economías de escala derivadas de la producción de grandes volúmenes.

- La utilización de mejoras técnicas.

- La relocalización en áreas que cuenten con factores productivos abundantes y baratos o subvencionado por las políticas públicas (suelo barato, exenciones fiscales).

- Otra forma de mejorar la competitividad es mediante la diferenciación de sus productos, lo cual permite obtener mayores utilidades (dado el mayor valor agregado). Para ello, es necesario que las empresas introduzcan mejoras en la calidad de sus materias primas, incrementen sus niveles de especialización, tengan mano de obra altamente cualificada e inviertan en investigación y publicidad. Por ello, Porter señala la necesidad de entender que las empresas no se encuentran aisladas, sino que existe una serie de proveedores, distribuidores y clientes integrados.

${ }^{14}$ Ibidem, p. 72. 
Los clústeres son fundamentales en el análisis de Porter. Los define como: "concentraciones geográficas de empresas interconectadas, suministradores especializados, proveedores de servicios, empresas de sectores afines e instituciones conexas que compiten pero que también cooperan". ${ }^{15}$

La diferencia de los clústeres con las economías de aglomeración se genera en que éstos buscan potenciar las concentraciones emergentes de empresas y apoyar el desarrollo de aquellas áreas que tengan relaciones más fuertes con cada clúster o mayores efectos sobre él. Los clústeres constituyen una multi organización y tienen una importante influencia en la competencia, las empresas, el Estado y las instituciones.

La importancia de los clústeres en la competencia radica en que éstos ofrecen ventajas en cantidad y costo; calidad y especialización de los factores; presencia de proveedores capacitados y sectores afines competitivos; clientes locales informados y exigentes; demanda local inusual; contexto local que fomenta formas adecuadas de inversión y mejora continua, así como una competencia fuerte entre las empresas locales.

Porter señala que en el corto plazo pueden aumentar las exportaciones de un país, dado el bajo costo de la mano de obra o la explotación de los recursos naturales con tecnología importada, pero esto sólo en el corto plazo. Para que una zona llegue a ser productiva, es necesaria la formación de clústeres. De lo contrario, no se podrá contrarrestar el incremento natural de los costos y, por tanto, las regiones que tengan mayores subvenciones o costos más bajos atraerán a las empresas, y esa no es la respuesta que requiere el contexto económico actual.

\section{ORIGEN, EVOLUCIÓN E IMPORTANCIA DE LA INDUSTRIA DE CALZADO EN LEÓN}

La ciudad de León se ubica al noroeste del estado de Guanajuato, colinda al oeste con el estado de Jalisco, al norte con el estado de San Luis Potosí, al este con el estado de Querétaro y al sur con el estado de Michoacán.

León es la ciudad con el mayor número de habitantes de la zona y tiene un límite urbano de aproximadamente 20000 hectáreas. El municipio tiene una extensión territorial de $1219.6 \mathrm{Km}$. Tiene la mayor participación municipal en el plano estatal de Guanajuato, con 35.5\% del PIB.

La ciudad de León tiene como característica primordial la producción de calzado. ${ }^{16}$ Es productor desde el año 1645 y se constituye como gremio

\footnotetext{
${ }^{15}$ Ibidem, p. 203.

${ }^{16}$ Según el directorio empresarial del gobierno de León, se cuenta con cuatro asociaciones de calzado, con una cámara de la industria de la curtiduría y, con 39 empresas de calzado, entre las cuales se encuentran empresas de diseño, de suajes, de fabricación de calzado, de insumos, de seguridad industrial, de refacciones y maquinaria para la industria.
} 
a partir de los años 1808 y 1809. ${ }^{17}$ "Desde la tercera década del siglo XIX comenzaron a procesarse insumos talabarteros para proveedores de aperos, como productos indispensables usados en su gran mayoría para el transporte. Sin embargo, la industria curtidora leonesa comienza a desarrollarse formalmente a principios del siglo xx."18

La industria del calzado se consolida al pasar a una etapa artesanal y lograr que la producción se realizara mayormente con insumos mexicanos. Lo anterior fue gracias a que durante la Segunda Guerra Mundial los dueños del capital extranjero se dedicaron a vender el calzado militar para Estados Unidos. Además, sus inversiones se destinaron a otros sectores con alto nivel tecnológico. ${ }^{19}$

A lo largo de la primera mitad del siglo xx, la gente dejó sus antiguas actividades y se concentró en la industria del cuero y el calzado, posiblemente porque la expansión del mercado ofrecía mejores oportunidades a las personas. Ello resultó de la acelerada industrialización y urbanización del país, del arribo de tecnología y las mejores remuneraciones que ofrecía dicha industria. ${ }^{20}$

Durante los años sesenta se crearon asociaciones de productores de calzado, así como nuevas fábricas. Con ello, se fortaleció la producción, sin embargo, se presentaron varias crisis económicas nacionales (1982, 1995, 2008 y 2009) que obligaron a muchas empresas a cerrar. No obstante, León se mantiene como la principal ciudad productora de calzado dentro de México y representa una de las principales industrias de calzado en el entorno mundial.

En el municipio de León la producción de calzado en el año 2014 fue de 135 millones de pares, cifra que equivale a 53\% de la producción nacional del calzado. El sector cuero-calzado contribuyó con 53.4\% de las exportaciones del municipio, en 2013. Así, la producción tradicional de cuero-calzado es uno de los pilares económicos de León.

En León, el mercado laboral está integrado por 622346 personas, de las cuales 93.6\% son personas ocupadas (582 295 trabajadores); sólo 6.4\% son personas desocupadas. ${ }^{21}$ Del total de la PEA ocupada, 294723 trabajadores $(50 \%)$ ganan de uno a tres salarios mínimos; es decir, de 2400 a 7200 pesos al mes. El promedio de años de escolaridad de la PEA es de 9.4 años. $^{22}$

\footnotetext{
${ }^{17}$ Martínez, Adriana, “¿Es factible hablar de un distrito industrial del calzado en León?”, Economía Informa, núm. 345, p. 147.

${ }^{18}$ Iglesias, Esther, La industria del cuero y el calzado en México, México, UNAM, 1998.

${ }^{19}$ Ibidem, p. 34.

${ }^{20}$ Martínez, Alberto, André, Ortiz, "Factores de competitividad, situación nacional y cadena productiva de la industria del calzado en León Guanajuato", Economía Sociedady Territorio, núm. 7, p. 550.

${ }^{21}$ Con base en datos del INEGI del primer trimestre del 2015, en México la población económicamente activa asciende a 52 millones de personas, de las cuales 49.8 millones se encuentran ocupados y 2.2 millones se encuentran desocupados.

${ }^{22}$ De la población ocupada en el estado, 1.1 millones de empleados ganan hasta un salario mínimo, 387 mil personas ganan más de uno y hasta dos salarios mínimos, 146 mil personas ganan más de 2 y hasta 3 salarios mínimos, y 86 mil personas ganan más de 3 y hasta 5 salarios mínimos.
} 
La industria del cuero-calzado continúa siendo la más importante en la producción del estado, representa 22\% del PIB del estado, las exportaciones representan $60 \%$ y el empleo $20 \%$. De las empresas existentes, $96 \%$ son micro, pequeñas y medianas empresas, y generan 64\% del empleo de la población ocupada dentro del municipio.

\section{MetOdOLOGía PARA LA inVEStigaCión \\ EN EL ESTUDIO DE CASO}

Consideramos que la entrevista es un instrumento más apropiado para tratar el tema de estudio, en virtud de la interacción personal y la mayor profundidad cualitativa que se obtiene, particularmente al entrevistar a los directores de las empresas. ${ }^{23}$

La segunda interrogante fue decidir si la entrevista sería estructurada o no, el formato (cerrado o abierto) y el orden de las preguntas. Existen argumentos a favor y en contra de ambos tipos de entrevistas. La primera es más apropiada para probar hipótesis específicas y cuantificar con rigor los resultados derivados de los diferentes interlocutores. ${ }^{24}$

Las entrevistas programadas, señalan algunos investigadores, facilitan el manejo y el análisis de los datos y reducen la probable parcialidad en entrevistas menos estructuradas, así como el riesgo de obtener excesivo material irrelevante. ${ }^{25}$ Por otra parte, las entrevistas no estructuradas proporcionan información más cualitativa y detallada sobre asuntos complejos. Existe consenso, sin embargo, en que una entrevista completamente no estructurada, sin dirección, es inapropiada para la investigación, dado que incrementa enormemente el trabajo de análisis. Así, un enfoque semiestructurado es más adecuado. ${ }^{26}$

Se consideró que no se debía divorciar una entrevista estructurada orientada más a lo cuantitativo de una no estructurada que privilegiara lo cualitativo. En algunos casos ambas se complementan se necesitan. Es el problema lo que determina el método y no el procedimiento contrario.

Las entrevistas pueden ser guiadas y todavía centrarse en grandes tópicos de interés. "La investigación sería más eficaz si los procedimientos permitieran a los entrevistados expresar sus propios puntos de vista al tiempo que proporcionan información lo suficientemente estructurada para el análisis

\footnotetext{
${ }^{23}$ Chadwick, Bruce, et al., Social Science Research Methods, New Jersey, Prentice Hall, 1984.

${ }^{24}$ Ibidem.

${ }^{25}$ Mitchell, Robert, "Survey Material Collected in the Developing Countries: Sampling, Measurement, and Interviewing. Obstacles to Intra an International Comparisons", International Social Science Fournal, núm. 17, pp. 204-226.

Somer, Robert, Somer, Barbara, A Practical Guide to Behavioral Research. Tools and Techniques, Nueva York, Oxford University Press, 1986, pp. 68-75.

${ }^{26}$ Kane, Eileen, "Basic descriptive research in the social sciences and humanities", en Eileen Kane, Doing your own research, London, Marion Boyars, 1983, pp. 35-40.
} 
y el informe sistemático." 27 Por ello, en la investigación se usó un formato semiestructurado que incluyó preguntas específicas acerca de tópicos de interés previamente determinados.

Las entrevistas fueron conducidas personalmente e incluyeron preguntas centradas en los asuntos investigados. Se dirigieron a personas con posiciones directivas en las empresas tomadas como muestra. En algunos casos, se dio la opción de que la entrevista se aplicara a mandos medios.

Las preguntas se ordenaron de menor a mayor importancia. Existe consenso de que, en general, las preguntas interesantes y relativamente fáciles de contestar deberían aparecer al principio. Una vez que se ha establecido cierta confianza. ${ }^{28}$

A los empresarios les gusta hablar de cómo empezaron sus compañías, de las condiciones que los llevaron a establecerse en una localización o relocalización y, con frecuencia, de manera inadvertida, de la acción alternativa que hubieran tomado si no tuvieran en el lugar establecido las condiciones para desarrollar su trabajo. Una vez establecido cierto ambiente de confianza, se pueden formular las preguntas más importantes y sensitivas.

Para vincular los elementos de la parte empírica con los elementos teóricos, se decidió realizar el trabajo de campo a través de una serie de quince entrevistas a empresas de la industria del calzado, ubicadas en el municipio de León, Guanajuato. Particularmente se estudiaron empresas con el mayor número de años establecidas en la ciudad de León. Se determinó entrevistar a cuatro empresas micro, seis empresas pequeñas, tres empresas medianas y dos empresas grandes.

Las quince entrevistas se realizaron de enero a abril de 2016, ello a través de un muestreo aleatorio con una confiabilidad de $85 \%$ y con un margen de error de $12.5 \%$. La fórmula para determinar el tamaño de la muestra fue la del universo conocido:

$$
\mathrm{n}=\frac{N \cdot Z^{2} \cdot p \cdot(1-p)}{(N-1) \cdot \mathrm{e}^{2}+Z^{2} \cdot p \cdot(1-p)}
$$

Donde:

$\mathrm{n}=\mathrm{El}$ tamaño de la muestra que queremos calcular.

$\mathrm{N}=$ Tamaño del universo.

$\mathrm{Z}=$ Es la desviación del valor medio que aceptamos para lograr el nivel de confianza deseado.

$\mathrm{e}=$ Margen de error máximo admitido.

$\mathrm{p}=$ Proporción que se espera encontrar

\footnotetext{
${ }^{27}$ Brown, J., Canter, D., "The uses of explanation in the research interview", en M. Brenner, et al., The research interview, uses and approaches, New York, Academic Press, 1985, p. 83.

${ }^{28}$ Somer, op. cit. pp. 35-48; Chadwick, op. cit. pp. 105-125.
} 
Con base en el uso de la metodología anteriormente señalada, se obtuvieron los resultados presentados en el siguiente apartado.

\section{LA INDUSTRIA DEL CALZADO EN LEÓN: VINCULACIÓN ENTRE LO TEÓRICO Y LO EMPÍRICO}

Los datos que se detectaron son sólidos para determinar un vínculo entre los enfoques teóricos revisados en la sección dos y los fenómenos empíricos descubiertos dentro del trabajo de campo.

La teoría de la localización señala que cuando una industria ha escogido una localidad para situarse en ella, existen grandes posibilidades de que permanezca en ese lugar durante largo tiempo. Ello resulta de las ventajas que se obtienen cuando las empresas se encuentran cercanas entre sí. En ese sentido, la ciudad de León se mantiene como productora de calzado desde 1645. En este momento, es la ciudad más importante a nivel nacional por valor de producción y exportación de calzado.

Con base en las entrevistas, los empresarios de calzado en León manifestaron que escogieron esa ciudad para producir calzado por dos principales razones: la existencia de proveedores cercanos y la facilidad para contratar mano de obra calificada. Este último punto es resultado de la tradición histórica para producir calzado en dicha ciudad.

En el estudio de referencia, se les preguntó a los entrevistados cuál hubiera sido el resultado de haberse ubicado en una ciudad diferente. La respuesta de todos fue "el fracaso". Dos de las empresas entrevistadas señalaron que hace muchos años iniciaron y consolidaron su empresa en la ciudad de México, tenían sus compradores en la capital del país, sin embargo, decidieron relocalizarse en León.

El objetivo para los dos empresarios fue reducir costos; dieron prioridad a la cercanía de proveedores y de mano de obra, antes que al aumento en los costos de transporte para llevar sus productos al mercado más grande del país: la Ciudad de México. Lo anterior se enmarca en las aportaciones teóricas vertidas por Marshall, quien argumenta que, cuando las empresas se aglomeran, se forma un mercado de trabajo especializado.

Sanfilippo y Adnan Seric ${ }^{29}$ se remiten a diferentes estudios ${ }^{30}$ para demostrar que la aglomeración en la producción del calzado en León representa

\footnotetext{
${ }^{29}$ Sanfilippo, Marco, Seric, Adnan, Spillovers from agglomerations and inward FDI. A Multilevel Analysis on SSA domestic firms, p. 20. [Consulta: 1 de julio, 2015]. Disponible en: http://papers.ssrn.com/sol3/ papers.cfm?abstract_id=2461195

${ }^{30}$ Véase Unger, Kurt, "Los clústeres industriales en México: especializaciones regionales y la política industrial", Documento de Trabajo, CEPAL. [Consulta: 17 de julio, 2015]. Disponible en: http://www. eclac.org/ddpe/noticias/paginas/8/15078/KurtUnger.pdf; Martínez, op. cit.; Martínez y Ortiz, op. cit.
} 
un clúster. ${ }^{31}$ En el trabajo de campo, se observa una integración de la cadena industrial en la cual se vinculan las materias primas y otros insumos para la producción. Se observa la integración de variadas empresas, destacan la industria textil, la de tintes y pinturas de madera, de proveedores de maquinaria y equipo, la industria ganadera, la de proveedores de hule, entre otras. Todas estas empresas son apoyadas por instituciones financieras educativas y de gobierno. Como argumentan algunos autores, la eficiente integración en cualquier cadena de abastecimiento es fundamental para asegurar la eficiencia y calidad de los productos elaborados.

Para el análisis de la cadena industrial se revisan varios niveles: $a$ ) Industrias del calzado y proveedores directos; $b$ ) Integración entre las empresas curtidoras, partes y componentes con sus proveedores, y c) Relación entre los fabricantes del mismo ramo. Con base en el trabajo de campo, se observa que sí existe integración de la cadena industrial, sin embargo, ésta es insuficiente, particularmente por la cadena de abastecimiento.

Con base en nuestras entrevistas, detectamos que las empresas de la industria del calzado en León se proveen con $42 \%$ de insumos locales. El resto lo adquieren de otros lugares del país o inclusive del extranjero. Cabe matizar que $80 \%$ de los proveedores de la curtiduría y de la proveeduría colaboran con las empresas del calzado para la mejora de los productos, apoyan con técnicas de producción y en el financiamiento. Sin embargo, se destaca que estos vínculos son informales y poco frecuentes.

De los entrevistados, $73 \%$ considera la relación entre empresas contratistas y maquiladoras como informal y esporádica. Esto es, dentro de los esquemas de subcontratación no existe fortaleza en la integración.

Por otra parte, $60 \%$ de los productores del calzado afirmó que deben pagar en efectivo la piel, porque el crédito para adquirir los insumos es escaso. Además, si existe un pedido grande por parte de una empresa productora de calzado, la proveeduría no siempre puede surtirla.

Martínez ${ }^{32}$ destaca que para el funcionamiento de un distrito industrial son importantes los flujos de comunicación. Para Marshall las externalidades generan tres fenómenos distintos. El primero es los flujos de información. En este punto, se observa que los productores de calzado de empresas pequeñas y medianas tienen nula o escasa comunicación, lo cual es resultado de los celos profesionales y de la competencia desleal. Los resultados de las entrevistas muestran que sólo 19.9\% de los entrevistados tiene contacto con otras empresas de la industria.

\footnotetext{
${ }^{31}$ Martínez, op. cit. hace una diferenciación, destaca que el clúster en la producción de Calzado en León, es un clúster natural.

${ }^{32}$ Martínez, op. cit., pp. 154-156.
} 
En relación con la comunicación entre proveedores y empresas, se logra apreciar que las grandes compañías tienen una estrecha comunicación con sus proveedores, pues $100 \%$ de los entrevistados tocó temas vinculados con la mejora del producto a través de la mejora en la proveeduría.

La escasa comunicación entre empresas de la misma industria nos lleva a observar en el sector de estudio una limitante para avanzar en el proceso de consolidación y expansión de las economías de localización. Henderson $^{33}$ plantea que las líneas de comunicación entre agentes que vinculan conocimiento toman tiempo para madurar y ser eficaces, incluso aunque éstas sean innovaciones menos sofisticadas. Por tanto, generar una red eficaz de intercambio de información no resulta fácilmente trasladable en el espacio.

Sin embargo, el autor matiza que el aprovechamiento de este tipo de externalidades genera la persistencia de clústeres sectoriales en determinadas localizaciones y, de ese modo, favorece la aglomeración de la actividad en el territorio. Bajo esta lógica, empresarios y Gobierno del estado de Guanajuato deben actuar para generar estrategias de comunicación entre los diferentes actores de la industria de calzado en León.

Las empresas de calzado en León tienen la posibilidad de diseñar su propio calzado, no obstante, contratan centros de diseño externo. Tal es el caso del Centro de Innovación Aplicada en Tecnologías Competitivas (Ciatec), el cual funciona desde 1976 en la ciudad de León. Su objetivo es "Proveer a las industrias del calzado, curtiduría y su proveeduría de los servicios tecnológicos que les apoyen para fortalecer sus ventajas competitivas a fin de que puedan afrontar con oportunidad y eficacia a sus competidores". ${ }^{34}$

El diseño no es el más adecuado para lograr competir en el mercado internacional, y es que, al existir muchas empresas del tipo artesanal, la adaptación a los estándares que exige la competencia mundial no ha sido rápida ni eficiente.

En el trabajo de campo se les preguntó a los empresarios si tienen al interior de las compañías expertos en innovación y diseño. Solamente 13.3\% sí tiene; el resto no. Las empresas ven como mejor opción contratar los diseños del Centro de Innovación Aplicada en Tecnologías Competitivas, resultado de la falta de personal capacitado en diseño e innovación.

Para Masahisa y Ogawa, ${ }^{35}$ las economías externas surgen al momento en que la producción aumenta, a medida que disminuye la distancia que va del

\footnotetext{
${ }^{33}$ Henderson, Vernon, Urban Development: Theory, Fact, and Illusion, Oxford, Oxford University Press, 1988. ${ }^{34}$ Centro de Innovación Aplicada en Tecnologías Competitivas (Ciatec), "Cientro de Innovación Aplicada en Tecnologías Competitivas", Emagister. [Consulta: 27 de febrero, 2016). Disponible en: http: //www. emagister.com.mx/ciatec-centrodetalles-45085.htm

${ }^{35}$ Masahisa, Fujita, Ogawa, Hideaki, "Multiple equilibrium and structural transition of non-monocentric urban configurations", Regional Science and Urban Economics, núm. 2.
} 
lugar de producción al mercado o al lugar de los insumos. La producción de calzado en León tiene las dos ventajas: la cercanía con la demanda final y con los insumos, y es que se encuentra cerca de ciudades con fuerte desarrollo industrial y, por ende con alto nivel adquisitivo: Celaya, Aguascalientes y Querétaro. A su vez, tiene una fuerte cercanía con los insumos, dados los muchos años de experiencia en el ramo que tiene la ciudad de León.

Para Ohlin, ${ }^{36}$ la distancia entre los recursos naturales y los mercados; el transporte de las materias primas y los productos acabados, y las diferencias locales en los recursos son lo que, ceteris paribus, influye en la localización de una industria en un lugar determinado. Precisamente, esos fueron los elementos que $100 \%$ de los empresarios entrevistados nos respondieron frente a la pregunta de las razones por las cuales escogieron León para desarrollar su industria.

En la ciudad de León destaca la existencia de una fábrica que se localizó originalmente en la Giudad de México, la cual tenía a sus compradores principalmente en la capital del país, sin embargo, decidió relocalizarse en León. El objetivo para ellos fue reducir costos y, por ende, dieron prioridad a la cercanía de proveedores y de mano de obra antes que a la cercanía con el mercado original. En ese sentido se confirma el argumento teórico de Fujita Masahisa y Thisse ${ }^{37}$ quienes afirman que el centro comercial puede surgir del centro del mercado. Por eso no se requiere que la industria esté ubicada en el punto donde se minimicen los costos de transporte.

Con base en el trabajo de campo, se logró observar la inexistencia de instituciones especializadas en la comercialización de zapatos, lo cual representa una debilidad en la industria. Aunque es una debilidad del clúster, se puede y debe convertir en una fortaleza, a partir de la instauración de instituciones especializadas en la comercialización nacional e internacional de calzado.

La aglomeración genera el establecimiento de otras actividades que son complementarias. En este sentido, a partir de los años sesenta se observa en el municipio de León la creación de asociaciones de productores de calzado. Los servicios ofrecidos por esas asociaciones y cámaras de la industria del calzado son bien aceptados por los empresarios de la ciudad, sin embargo, no todos usan sus servicios. De los entrevistados, $20 \%$ no usa los servicios ofrecidos por las cámaras; el resto, sí considera que las cámaras les han generado beneficios a sus empresas.

La aglomeración forma un mercado de trabajo especializado, por tanto, se puede compartir con todas las empresas del sector. Empíricamente se

\footnotetext{
${ }^{36}$ Ohlin, Bertil, Comercio interregional e internacional, Barcelona, Oikos-tau, 1971.

${ }^{37}$ Masahisa, Fujita, Jacques-Francois, Thisse, Economics of Agglomeration: Cities, Industrial Location, and Globalization, Massachusetts, Cambridge University Press, 2013, p. 269.
} 
observa a lo largo de la primera mitad del siglo xx, en la ciudad de León, que la gente dejó sus antiguas actividades y se concentró en la industria del cuero y el calzado. Teóricamente, trabajadores y empresarios ganan con la aglomeración, pues se genera para el empresario una oferta de trabajo especializada y, por otra parte, los trabajadores tienen la seguridad de no depender solamente de una empresa para obtener empleo.

Con base en las entrevistas, detectamos que lo anterior tiene una validez intermedia para esta industria, y es que, para elaborar calzado, la destreza manual es el elemento fundamental. Por otra parte, el trabajo en la industria del calzado se va desmembrando cada vez más dentro del sector. Se detectó que para los empresarios lo anterior genera beneficios y retrocesos, pues cuando las empresas - en particular las pequeñas - quieren contratar mano de obra, los obreros se encuentran muy especializados. De este modo, es complicada la contratación, porque las empresas pequeñas buscan personas que conozcan todas las partes del proceso de producción.

Por otra parte, la especialización es benéfica para las empresas medianas y grandes porque les permite contar en cualquier momento con personal apto para el desempeño de una función particular. Además, estas empresas buscan mano de obra especializada en una parte del proceso de producción porque ello representa mayor eficiencia y eficacia. Con lo anterior se detectan ventajas y desventajas de la especialización de la mano de obra, lo cual es resultado de la necesidad de competitividad requerida por las empresas en el contexto del comercio internacional.

Dice Marshall que los misterios de la industria pierden el carácter de tales, por así decir, están en el aire y los niños aprenden mucho de ellos de un modo inconsciente. El buen trabajo es apreciado; los inventos y los perfeccionamientos en la maquinaria, en los procesos de fabricación y en la organización general de los negocios se estudian pronto para dilucidar sus méritos o inconvenientes. Si una persona lanza una nueva idea, ésta es adoptada por las demás y combinada con otras sugerencias. De este modo, una idea se transforma en fuente de nuevas ideas.

Empíricamente, y con base en el trabajo de campo, se observa un elemento importante en la productividad del sector calzado de la ciudad de León: la experiencia se origina a partir del aprender haciendo y del aprendizaje interactivo. En la región de estudio, la aglomeración del sector calzado ha proveído una serie de apoyos externos para las empresas localizadas en ese lugar. Por ejemplo, el municipio tiene instituciones que promueven la capacitación.

Un elemento importante en la productividad del sector calzado es la experiencia laboral. En las industrias tradicionales, ésta se origina al aprender haciendo y con el aprendizaje interactivo. Así, los trabajadores aprenden durante el proceso de producción o a través de los mismos compañeros de trabajo. 
Se observa que los más de 360 años de experiencia en la industria del calzado en León facilita la contratación de mano de obra calificada. Los empresarios manifestaron una alta rotación e insuficientes programas de capacitación laboral. Contrario a ello, la capacidad de los trabajadores en habilidades múltiples, capacidad para innovar y el aprendizaje fue positiva.

De los entrevistados, $100 \%$ considera que León es una ciudad con excelente ubicación geográfica. Los empresarios manifestaron que cuentan con una adecuada infraestructura: carreteras, aeropuerto, aduanas, infraestructura energética y telecomunicaciones. Además, se detecta la cercanía entre fabricantes, mercado, universidades e institutos de investigación y diseño.

Existen programas de apoyo federal y estatal para las exportaciones. Destacan los programas Drawback, Altex, Ecex, Pitex, Cofoce y los ofrecidos por Bancomext. Como resultado de las entrevistas, destaca que sólo 33\% de las empresas utilizan alguno de los programas ofrecidos por el gobierno. Los empresarios señalaron falta de confianza y algunos de los requisitos que no se pueden cumplir para ser beneficiados con los programas.

Para que una economía sea de urbanización, son necesarias las siguientes variables: el acceso a un mercado más grande; el desarrollo de los mercados de mano de obra; la presencia de servicios comerciales de banca y financieros; transportes y medios de comunicación eficientes; servicios especializados (contables, consultores de empresas y firmas de publicidad); existencia de instalaciones sociales, culturales y de ocio; fabricantes de componentes; centros de consumo; universidades e institutos.

Los elementos antes señalados se observan en León Guanajuato, por ende, podemos afirmar que en esta región se manifiesta una economía que, en sentido teórico, representa una economía de urbanización. Además, en dicho lugar se integran diferentes empresas, entre las cuales destacan la industria textil, la de tintes y pinturas de madera, de proveedores de maquinaria y equipo, la industria ganadera y de proveedores de hule.

\section{REFLEXIONES Finales}

En el marco de la globalización y de los nuevos procesos de competencia basado en las nuevas tecnologías, ha renacido el interés por analizar y estudiar la localización de las actividades industriales y el éxito que éstas han tenido en los países de ingresos altos, particularmente cuando se logran conformar clústeres industriales. Precisamente la reflexión anterior motivó el desarrollo de esta investigación. Por otra parte, es importante la industria del calzado en León como estudio de caso, pues es una industria generadora de empleos, de ingreso y de divisas en la zona centro del país, además de uno de los clústeres más importantes del país. 
El trabajo partió del análisis de la teoría de la localización y de la aglomeración. Se consideraron autores clave en la teoría industrial, como Marshall, Weber, Krugman y Porter. Posteriormente, con el trabajo de campo, los empresarios entrevistados señalaron que sus principales incentivos para ubicarse en León fueron reducir costos y darle prioridad a la cercanía de proveedores y de mano de obra, antes que al aumento en los costos de transporte para llevar sus productos al mercado más grande del país: la Ciudad de México.

Se observó la existencia de un clúster de calzado en León, pues se integra la cadena industrial donde se vinculan las materias primas y otros insumos para la producción. Por otro lado, aunque se detectó una comunicación no muy estrecha entre proveedores y empresas, ésta se puede mejorar con estrategias de política pública y empresarial.

La aglomeración en León ha generado el establecimiento de otras actividades que son complementarias. En este sentido, a partir de los años sesenta se observa en el municipio de León la creación de asociaciones de productores de calzado.

Con base en el trabajo de campo, se observó un elemento importante en la productividad del sector: la experiencia se origina a partir de aprender haciendo y del aprendizaje interactivo. Durante estos procesos, los trabajadores aprenden en la producción y con los mismos compañeros de trabajo.

Las empresas de calzado en León se encuentran rodeadas de economías externas y de aglomeración, lo cual se observa con los servicios existentes, aunados a las estructuras sociales que se originaron en la ciudad de León desde el año 1800. Más de 360 años de historia han generado experiencia en la mano de obra, en la adquisición de insumos, en transporte y en la venta, lo cual, finalmente, representa una ventaja competitiva que se debe aprovechar y preservar.

Detectamos que las empresas grandes establecen relaciones de subcontratación con las pequeñas, sin embargo, para que funcione, se requiere el cumplimiento de estándares de calidad, compromiso con la entrega, diseños modernos, entre otras condiciones. Para lograr lo anterior, se debe generar un vínculo a través de la asistencia tecnológica, financiera y de trasmisión de conocimiento.

Cada uno de los puntos señalados muestran la vinculación estrecha entre la postura teórica y empírica. Por ello, podemos afirmar que en dicho sector se hacen presentes economías de localización y de aglomeración. Esto aunado a la existencia de un clúster que se encuentra en proceso de consolidación, pero con grandes ventajas para permanecer en la esfera del comercio nacional e internacional.

Se recomienda una participación más activa de las instituciones públicas, tanto del Gobierno del estado de Guanajuato como del Gobierno federal. 
Y es que es importante mantener y fortalecer el diamante que se ha venido formando a lo largo de los años, en esa importante región mexicana, a través de una política industrial más activa.

También es importante recalcar la necesidad de seguir fortaleciendo el clúster del calzado en León. Hoy, en el marco de la globalización, los países no compiten, compiten las empresas y las regiones. Por ello, se debe mantener la estrategia industrial y fortalecer la presencia del calzado mexicano en el mercado internacional. 